Urologe 2016 $55: 660$

DOI 10.1007/s00120-016-0071-z

Online publiziert: 4. März 2016

๑) Springer-Verlag Berlin Heidelberg 2016

CrossMark

J. Heß

Klinik und Poliklinik für Urologie, Uroonkologie und Kinderurologie, Universitätsklinikum Essen, Essen, Deutschland

\title{
STHLM3-Test könnte helfen, das Prostatakarzinomscreening zu verbessern
}

0,72-0,75) im Vergleich zum PSA-Wert alleine (mit einer AUC 0,56, 95 \% Konfidenzintervall 0,55-0,60). Alle Variablen des STHLM3-Modells waren nach multipler logistischer Regression signifikant mit dem Vorliegen des High-risk-Prostatakarzinoms assoziiert $(p<0,05)$. Im Vergleich zu einem PSA-Schwellenwert von $\geq 3 \mathrm{ng} / \mathrm{ml}$ konnte der Einsatz der STHLM3-Testbatterie bei gleicher Sensitivität zur Detektion von high grade Karzinomen die Anzahl der Biopsien um $32 \%$ (95\% Konfidenzintervall 24-39) reduzieren und $44 \%$ (95\% Konfidenzintervall 35-54) an "gutartigen“ Biopsien vermeiden.

Bei alleinigem Einsatz des PSA-Wertes sind bei $17 \%$ Biopsien mit Nachweis eines Gleason-Score 6-Karzinoms durchgeführt worden, welche im Studienmodell vermieden worden wären. Umgekehrt wiesen von den 603 High-risk-Karzinomen, die im STHLM3-Modell identifiziert wurden, 124 (21\%) einen PSAWert zwischen 1 und $3 \mathrm{ng} / \mathrm{ml}$ auf und wären durch alleinigen Einsatz des PSAWertes nicht biopsiert worden.

Auch hier wird die Zeit zeigen, ob sich diese aufwendige Testbatterie in den klinischen Alltag integrieren lässt. Die Ergebnisse zeigen jedoch, dass auch das Screening des Prostatakarzinoms zukünftig noch stärker individualisiert werden wird und Variablen jenseits der bekannten klinischen Parameter eine zunehmend wichtige Rollen einnehmen werden.

\section{Korrespondenzadresse}

\section{Dr. J. Heß}

Klinik und Poliklinik für Urologie, Uroonkologie und Kinderurologie, Universitätsklinikum Essen Hufelandstraße 55, 45147 Essen, Deutschland jochen.hess@uk-essen.de

Interessenkonflikt. J. Heß gibt an, dass kein Interessenkonflikt besteht. 